\title{
Vega and the local interstellar medium
}

\author{
D.A. Bohlender and R.E.M. Griffin \\ Herzberg Institute for Astrophysics, DAO, Canada
}

\begin{abstract}
Very high-quality, high-resolution optical CCD and photographic spectra of Vega reveal interstellar components in many spectra lines. The presence of these interstellar features suggests that detailed studies of such bright stars carried out with superb new data now available must take careful account of such contaminants in both abundance determinations and line profile fitting. Analyses of the interstellar features themselves can contribute to the general knowledge of the local ISM, an area previously largely limited to UV observations obtained by space facilties.
\end{abstract}

Keywords. Line: profiles, stars: early-type, stars: chemically peculiar, stars: indiviual: (Vega, Sirius), ISM: lines, techniques: spectroscopic

\section{Introduction}

It is a well-worn truism that the more you look, the more you see. How aptly that can be applied to the spectra of bright stars! The A-type stars, in particular, are plentiful and many are bright, and because they can be so easily observed they are superb resources for very detailed studies of the phenomena, especially the oddities, which are essential steps to progress in astrophysics.

IAU Colloquium 138 (Trieste, 1992) was entitled, "Peculiar versus Normal Phemonena in A-type and Related Stars", but it will be hard to stage such a meeting in the future because we are rapidly running out of "normal" A-type stars, partly because there are so many well-defined categories of abnormality, but also because the peculiarities are relatively easy to observe. Vega, once the archetypal stellar standard, is a good case in point.

\section{Asymmetries in lines of Vega}

Many years ago we were already aware of the curious flat-bottomed profiles of the lines in Vega (HD 172167), but had not fathomed the reason until the convincing modelling by Gulliver et al. (1991, 1994) (see also Hill et al. 2005) who demonstrated that Vega is very probably a rapidly-rotating star viewed pole-on. At that time our prime source of data was an unpublished high- $S / N$ stellar atlas of Vega and Sirius, derived from spectra at dispersions of $0.75-1.5 \AA \mathrm{mm}^{-1}$ recorded on fine-grain photographic plates at the Mount Wilson 100-inch coudé. Those low-noise spectra offered the advantage of very wide spectral coverage $(\lambda \lambda 2970-8500)$. We also knew that certain categories of lines showed more marked flattening than others, so our first investigation was to classify the profiles of all visible lines (some 880) in the spectrum of Vega according to the degree of flattening they exhibited.

\subsection{Lines of $\mathrm{Fe} \mathrm{I}$}

We selected 54 profiles of unblended Fe I lines in Vega, and separated them according to their central depths: $<2.5 \%$ (weak), from 2.5 to $7 \%$ (medium) and $>7 \%$ (strong), and formed the average of each sub-group. Those means are plotted in the upper panel of 

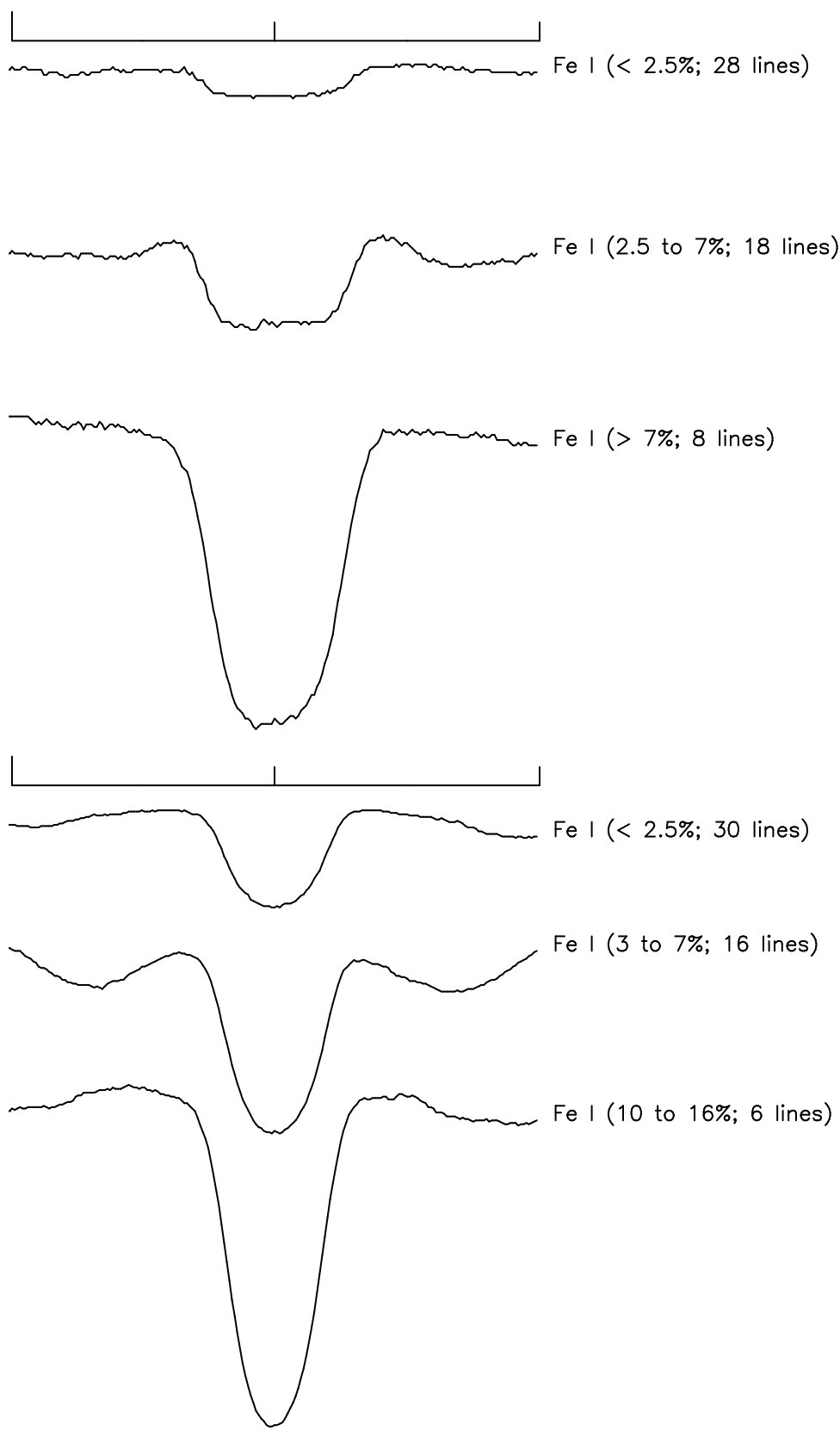

Figure 1. Averaged profiles of Fe I lines of differing strengths in Vega. (upper) and Sirius (lower).

Fig. 1, where we see for the first time not only the square profiles in the weak lines but also a blue-shifted asymmetry in the strong ones. The medium-strength profile also confirms another of our findings: the bottom is not strictly flat but is slightly concave. The latter may be caused by the superposition of a weak asymmetry upon an otherwise flat-bottomed profile, but it was prevalent enough that we classified a whole sub-set of 

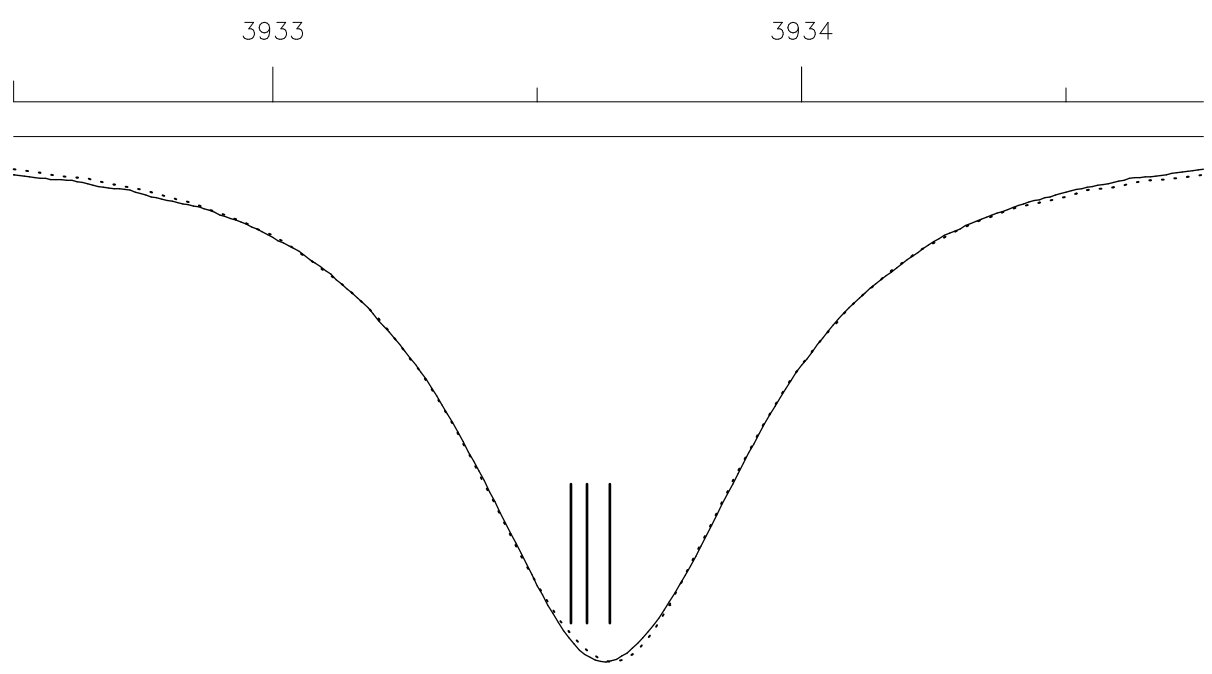

Figure 2. The Ca II K-line in Vega: forward (smooth line) and reversed (dotted line). The short vertical lines are the $L I C$ velocities.

lines on the basis of that central "reversal". A parallel investigation of 52 lines in Sirius (HD 48915) (Fig. 1) did not show any of those abnormalities.

\section{2. $C a$ II $\mathrm{K}$ line}

We also detected an asymmetry in the core of the Ca II K line, which we re-observed several times with the DAO 1.2-m telescope and high-dispersion $(R \sim 90000)$ coudé spectrograph and averaged to obtain high $S / N$. By reflecting the line profile about its mid-point (see Fig. 2) we could show that the asymmetry is due to extra absorption on the short-wavelength side. A similar test on the K line in Sirius showed no such asymmetry. The blue-shifted asymmetry in Vega was reported by Lallement et al. (1995) in Fe II $\lambda 2344$ from GHRST spectra; they attributed it to absorbers in the local interstellar cloud $(L I C)$, and modelled it as a combination of three components with velocities at $-12.7,-16.0$ and $-18.3 \mathrm{~km} \mathrm{~s}^{-1}$.

\section{CFHT Gecko spectra of Vega}

To investigate all three effects, we conducted follow-up spectroscopy of Vega with the echellette coude spectrograph Gecko on the CFHT, either directly or retrieved from the CFHT archive maintained and operated by the Canadian Astronomy Data Centre (CADC). This efficient, single-order instrument produces superb spectra with a resolving power of $100000-120000, S / N>1000$ (and in some cases substantially higher), yet with typical exposure times of only a few minutes.

Observations of the Na D lines (Fig. 3) confirm that the asymmetry is caused by a blue-shifted absorption, but that it is not present in all strong lines; it is not seen in the strong Mg II line at $\lambda 4481$.

Gecko spectra of other lines are reproduced in Figs. 4 and 5, where they show well the blue-shifted asymmetry, and also the dependence, implied in Fig. 1, of the line profile on ionization stage and excitation potential. Fig. 4 provides convincing confirmation that it is only the weakest lines in Vega which have the flat-bottomed profiles.

The short vertical lines in Figs. 2-5 indicate the positions of the LIC components. 


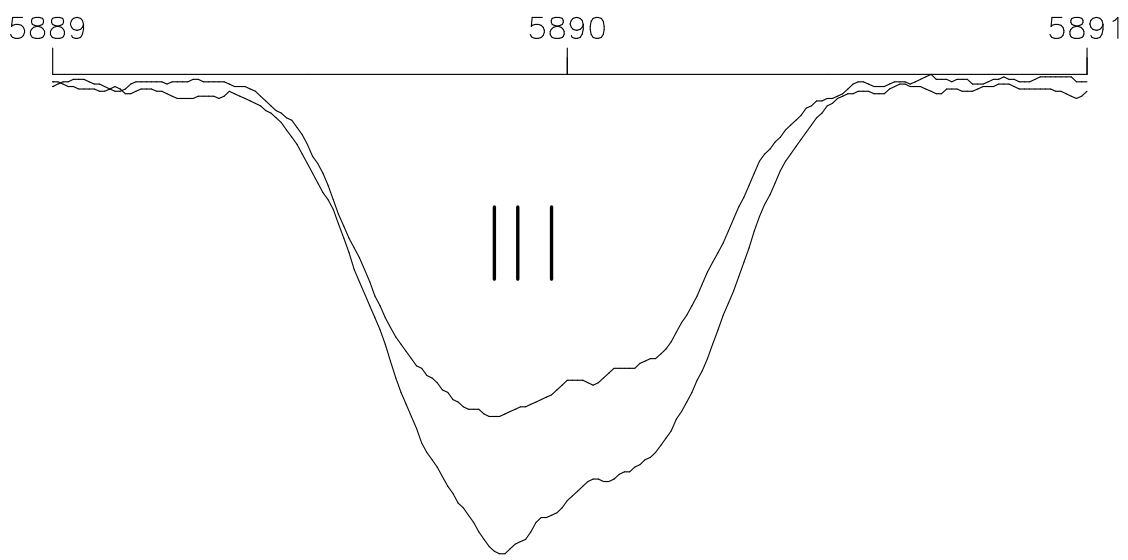

Figure 3. Gecko spectra of Na D1 and D2 in Vega. D1, the weaker line, has been shifted to the same wavelength as D2. The short vertical lines mark the velocities of the three $L I C$ components (Lallement et al. 1995) fitted to D2. (Telluric lines have been removed.)

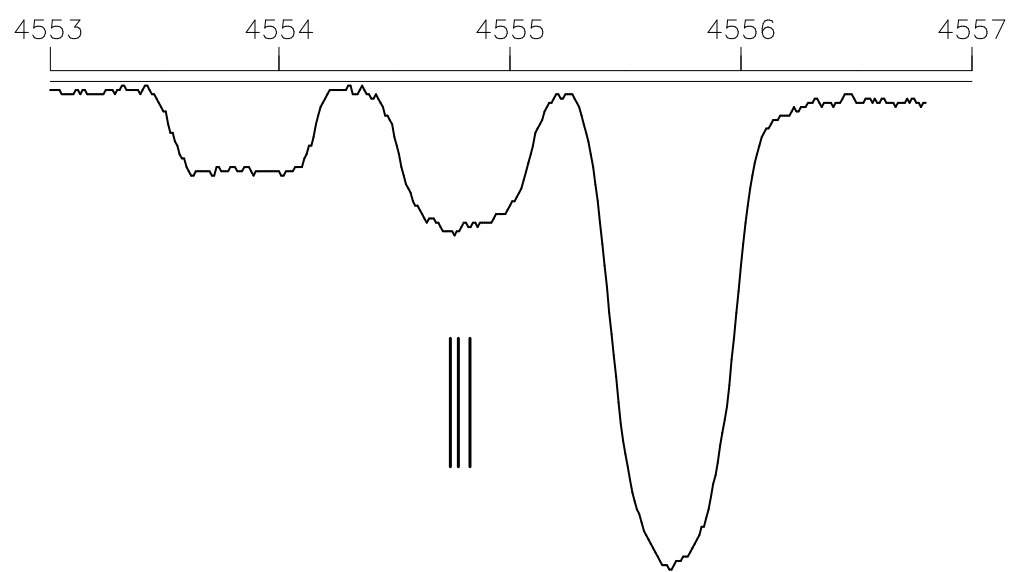

Figure 4. Gecko spectrum of Vega: $\lambda 4554.03$ (Ba II 1), $\lambda 4555.02$ (Cr II 44) and $\lambda 4555.89$ (Fe II $37)$.
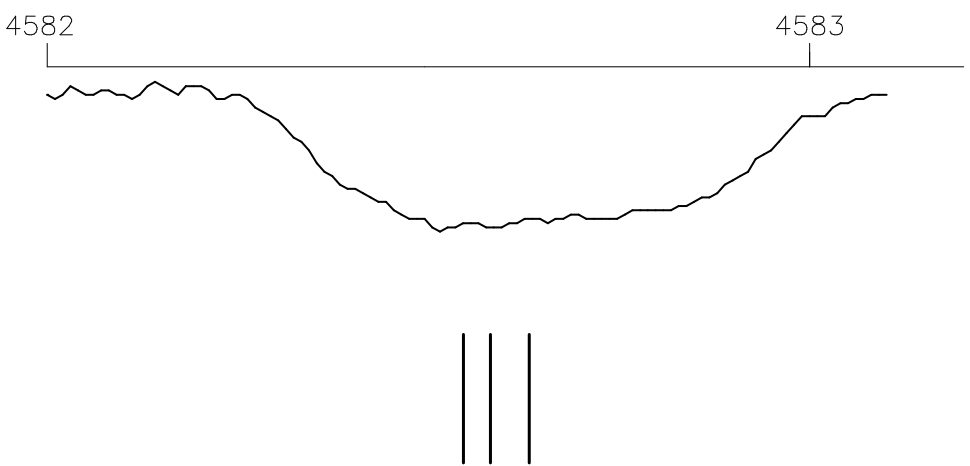

Figure 5. Gecko spectrum of Vega: $\lambda 4582.83$ (Fe II 37). 
In all cases there is a correspondence between the blend of the UV features and the blue-shifted asymmetries in the optical lines

\section{Conclusions}

This paper provides convincing evidence that the line profiles in the optical spectrum of Vega are slightly distorted by interstellar features (the $L I C$ absorption primarily occurs in low-EP lines, thus explaining its absence from certain strong stellar lines such as Mg II $\lambda 4481$ ). The magnitude of those distortions is sufficient to mask or interfere with modelling of the flat-bottomed profiles already reported. The dependence of "squareness" upon excitation potential indicates how Vega's rotational oblateness affects the constituents of its atmosphere differently, and is an aspect that accurate models of the rotation, and of the star itself, must predict. Since those same profiles are also affected differentially by non-negligible $L I C$ absorption, as shown above, that must be taken fully into account as well.

A more extended and rigorous study of these optical line asymmetries will also make a valuable contribution to our knowledge of their sites of formation in the $L I C$, a topic that has hitherto largely been limited to UV observations obtained from space.

Our ability to detect and measure $I S$ features in a well-observed star such as Vega demonstrates the need to make due allowance for similar effects in very high $\mathrm{S} / \mathrm{N}$ spectroscopy of other bright stars too.

\section{Acknowledgements}

REMG is grateful to the DAO and to the Symposium organizers for financial assistance to attend this meeting.

\section{References}

Gulliver, A.F., Adelman, S.J., Cowley, C.R. \& Fletcher, J.M. 1991, ApJ 380, 223

Gulliver, A.F., Hill, G., \& Adelman, S.J. 1994, ApJ, 429, L81

Hill, G., Gulliver, A.F., \& Adelman, S.J. 2005, These Proceedings, 35

Lallement, R. Ferlet, R., Lagrange, A.N., Lemoine, M. \& Vidal-Madjar, A. 1995, A $\& A$ 304, 461 